Cuad. Invest. Filol., 43 (2017), 33-49. http://doi.org/10.18172/cif.2974

\title{
ENJEUX DE L'ÉLOGE À JEANNE-MARIE LEPRINCE DE BEAUMONT DANS LES LETTRES SUR L'ANGLETERRE, LA HOLLANDE ET L'ITALIE D'ANNE-MARIE DU BOCAGE
}

\author{
María Isabel Corbí Sáez \\ Universidad de Alicante \\ maribel.corbi@ua.es
}

\begin{abstract}
RÉSUMÉ: Prenant en compte que l'«Amazone du Parnasse » selon les termes de Voltaire publia sa correspondance privée en vie et considérant que ce type de publications souffre des modifications de la part de leurs auteurs, notre article se propose de démontrer que la référence élogieuse à Leprince de Beaumont écrite par A.-M. du Bocage dans ses Lettres sur l'Angleterre, la Hollande et l'Italie n'est pas fortuite. Après avoir argumenté que cette référence fut introduite postérieurement en vue de la publication des lettres en 1764, ayant en tête les enjeux que du Bocage accorde au genre épistolaire, nous analysons la portée de l'élogieuse notation qui sans aucun doute déploie un jeu intertextuel. Notre étude nous amène à analyser l'intention de Du Bocage de se situer pour la postérité aux côtés de Leprince de Beaumont dans le débat essentiel et incontournable sur l'Éducation du XVIII ${ }^{2}$ siècle.

MOTS CLÉS: XVIII ${ }^{e}$ siècle français, A.-M. du Bocage, J.-M. Leprince de Beaumont, correspondances publiées, débat sur l'Éducation.
\end{abstract}

\section{DESAFÍOS DEL ELOGIO A JEANNE-MARIE LEPRINCE DE BEAUMONT EN LETTRES SUR L'ANGLETERRE, LA HOLLANDE ET L'ITALIE DE ANNE-MARIE DU BOCAGE}

RESUMEN: Teniendo en cuenta que la "Amazona del Parnaso" en términos de Voltaire publicó en vida su correspondencia privada, y considerando que este tipo de publicaciones sufría evidentes modificaciones por cuenta de sus autores, nuestro artículo se propone demostrar que la referencia elogiosa a Leprince de Beaumont escrita por A.-M. du Bocage en sus Lettres sur l'Angleterre, la Hollande et l'Italie no es fortuita. Tras primero dilucidar que esta referencia fue introducida posteriormente para la publicación de las cartas en 1764, y considerando los desafíos que du Bocage encuentra en el género epistolar, analizamos el alcance de la elogiosa anotación que, sin lugar a dudas, despliega un juego intertextual. Nuestro estudio nos lleva a evaluar la intención de du Bocage de situarse para la posteridad junto a Leprince de Beaumont en el esencial e ineludible debate sobre la Educación del siglo XVIII.

PALABRAS CLAVE: siglo XVIII francés, A.-M. du Bocage, J.-M. Leprince de Beaumont, correspondencias publicadas, debate sobre Educación.

Recibido: 29/02/2016. Aceptado: 02/11/2016 
Depuis ce temps-là, on a attaché presque autant de honte au savoir des femmes qu'aux vices qui leur sont défendus.

Mme de Lambert « Réflexions nouvelles sur les femmes »

\section{Introduction}

Les mots de Madame de Lambert avec lesquels nous commençons notre article démontrent combien de courage, d'assurance, de volonté et de hardiesse, il a fallu à nombre de femmes auteur qui ont défendu envers et contre tout, surtout sur les deux premiers tiers du XVIII ${ }^{\mathrm{e}}$ siècle, leur place de droit dans le débat d'idées propres aux Lumières aux côtés de leurs homologues homme. Quand nous abordons l'étude de la place que les femmes d'esprit ${ }^{1}$ occupent dans la circulation des savoirs à cette époque, Anne-Marie du Bocage et Jeanne-Marie Leprince de Beaumont en constituent bien deux cas incontournables. Il nous a semblé intéressant de présenter dans le cadre de notre texte les résultats d'une analyse au sujet de la présence de la célèbre pédagogue « avant la lettre » dans Les Lettres sur l'Angleterre, la Hollande et l'Italie d'Anne-Marie du Bocage. Les lecteurs et lectrices de cette correspondance publiée du vivant de leur auteure pouvant lire à la cinquième lettre du recueil: « [...] Madame Leprince de Beaumont fait de très bons traités d'éducation et les met en pratique [...]» (1770:49).

Un compliment, on peut l'imaginer, sincère, et surtout une reconnaissance indéniable à une femme auteur qui dans la mi-décennie des années 1760, comptait déjà avec une œuvre prolixe contenant les divers Magasins parmi tant d'autres. Un compliment et une reconnaissance, pouvons-nous penser, puisque ces femmes de lettres - toutes deux rouennaises, toutes deux catholiques - se sont également fréquentées lors du séjour d'Anne-Marie du Bocage en Angleterre en 1750. Pouvons-nous imaginer de même, en toute légitimité, que ce compliment et reconnaissance dérive de la gratitude de notre auteure pour avoir pu contribuer avec quelques-uns de ses textes poétiques au Nouveau Magasin Français².

1. Rappelons le caractère péjoratif des dénominations d' « intellectuelles », de « savantes » assignées aux femmes auteur du XVIII siècle. Nous retenons celle de « femme d'esprit » en suivant le propos et la définition que donnent Roland Bonnel et Catherine Rubinger (1994 : 9).

2. Ce périodique Nouveau Magasin Français ou Bibliothèque instructive et amusante dirigé par Jeanne-Marie Leprince de Beaumont est publié en langue française à Londres de 1750 à fin 1751. A.-M. du Bocage y publie dans le numéro d'octobre 1750 ses « Vers sur Renelash ». Voir à ce propos (Kaltz 2000 : 11). Ce périodique, l’un des premiers périodiques français dirigé par une femme (Kaltz 2000 : 9) se présente comme miscellanée de textes d'auteurs divers appartenant à des disciplines telles que " [...] l'Anatomie, Physique, Histoire, Droit alternant avec des anecdotes édifiantes, des considérations sur 
Pourtant, cet éloge nous amène quand même à nous poser quelques questions. N'est-il pas frappant qu'Anne-Marie du Bocage, célèbre salonnière, lauréate d'un prix littéraire ${ }^{3}$, membre de diverses académies faisant clairement exception à son époque (Bonnel et Rubinger 1994 : 20), amie de nombreux philosophes, saluée par l'élite lettrée masculine, ait pu vanter l'œuvre de Beaumont tout en sachant que le cénacle masculin a bien souvent sous-estimé, voire même dénigré la célèbre gouvernante, comme il est fort connu. Retenons tout simplement à l'esprit, comme exemple, le qualificatif de "magasinière ${ }^{4}$, en l'occurrence ironique, versés à plusieurs reprises par Voltaire - ami et admirateur de du Bocage - ; un qualificatif dépréciatif en toute évidence. Donc, un compliment et une reconnaissance qui retient notre intérêt, d'autant plus qu'aucune autre femme auteur contemporaine française n'est mentionnée dans ces lettres. Par ailleurs, nous nous devons de signaler qu'à l'époque de la première publication des Lettres sur l'Angleterre, la Hollande et l'Italie - en 1764 -, les appréciations directes sur la valeur d'une œuvre de la part d'une femme auteur envers une autre sont extrêmement rares. Il faudra attendre la dernière partie du XVIII ${ }^{e}$ siècle et le début du XIX ${ }^{\mathrm{e}}$ pour voir ce phénomène critique avec, par exemple, Madame de Genlis (Reid 2007 : 12-13).

Notre analyse de la présence de cette référence à cette pédagogue et femme de lettres, amplement célébrée à son époque dans l'Europe entière selon Barbara Kaltz (2000 : xviii), et, tel que nous l'avons souligné précédemment, ardemment critiquée dans son propre pays dans le camp des philosophes, est justifiée car la critique, y compris à l'heure actuelle, s'est simplement limitée à relever cette référence dans la correspondance de du Boccage ${ }^{5}$, mais sans avoir analysé les

l'actualité, des extraits d'auteurs contemporains, en vers ou en prose, un feuilleton inédit de Marie Leprince de Beaumont qu'elle publiera plus tard sous la forme d'un roman par lettres. Les deux correspondants les plus réguliers pour la partie scientifique sont Le Cat et Haller » (Artigas-Menant 1991 : 346).

3. Voir à ce propos l'intéressant article de John Iverson et Marie Pascale Pieretti, " "Toutes personnes $[\ldots]$ seront admises à concourir”: la participation des femmes aux concours académiques » (2004 : 313-332).

4. « Voltaire peut même sembler aimable en apparence du fait de l'ironie. Certains auteurs sont beaucoup plus incisifs dans leurs depreciations : Grimm, par exemple, qui parlant de L'éducation complète voyait son manuel d'éducation bon pour des “perroquets” » (in Kaltz 2000 : 24).

5. Déjà Grace Stuart Gill Mark relève cette référence dans la première étude critique sur A-M. du Bocage publiée en 1927 (1927 : 62). Parmi les critiques qui de nos jours signalent ceci, nous citons, par exemple, Jean-Marie Robain. Dans son œuvre Madame Leprince de Beaumont intime. Avec ses principaux contes et des documents inédits, il fait référence à la rencontre de du Bocage et de Leprince de Beaumont et à la notation dans les Lettres anglaises [sic] (2004 : 45). Cette référence a été signalée, certes, mais sans jamais avoir été analysée. 
raisons de cet éloge et sa portée. Ce à quoi nous dédions les pages de notre article.

Nous trouvons un intérêt tout particulier à la publication de cette correspondance par décision et choix personnels du vivant de leur auteure ${ }^{6}$ puisque, tel que le souligne Janet Gurkin Altman (1992 : 623), ce n’est qu’à partir des années 1760 que se donne la publication de ce type de correspondances privées. Mais à savoir, celles des philosophes hommes. Publiées par les épistoliers mêmes, il est fort admis que leurs lettres aient subi des modifications de leurs plumes, et ces modifications ne sont pas fortuites en toute évidence. Donc, dans le cas qui nous occupe, retenant qu'en mai 1750, Anne-Marie du Bocage rencontre en Angleterre Madame Leprince de Beaumont à une réception chez « Mylady Montaigu », on peut supposer qu'elle dut en faire part à sa sœur de façon immédiate, les lettres ayant été initialement adressées à sa cadette, tel qu'indiqué dans le paratexte de leur publication (1770 : Aij). Pourtant, nous soutenons sans risque d'erreur, nous semble-t-il, que d'une toute autre façon, puisqu'à cette date-là Madame Leprince de Beaumont n'avait pas encore publié de traités d'éducation ${ }^{7}$. Par conséquent, il n’est pas osé déduire que ce compliment et cette reconnaissance furent introduits de la sorte en toute intention dans le texte définitif en vue de sa première publication en 1764 .

Notre article se propose donc d'analyser, comme annoncé, les enjeux de la présence de Madame Leprince de Beaumont dans Les lettres sur l'Angleterre, la Hollande et l'Italie. Dans un premier temps, nous analysons comment Anne-

6. La plupart des femmes se vouant à la littérature, fuyant la honte et le dénigrement infligés par leur société, se virent obligées de publier leurs ouvrages dans l'anonymat pratiquement sur tout le dixhuitième siècle. Voir à ce propos l'« Introduction » au volume collectif édité par Roland Bonnel et de Catherine Rubinger, Femmes savantes et femmes d'esprit (Bonnel et Rubinger 1994 : 14). Anne-Marie du Bocage a publié sous son nom et de son vivant des œuvres de création qui s’inscrivent dans différents genres (Les amazones en 1748 - tragédie -, Colombiade ou la foi portée au nouveau monde en 1758, poème épique -, des poèmes de circonstances qu'elle recueille dans ses œuvres complètes, etc.), outre ses nombreuses traductions de l'anglais au français (voir par exemple la traduction adaptation du Paradise lost de Milton) et la correspondance qui nous occupe dans le présent article. Ses œuvres ont été traduites dans de nombreuses langues. Voir à ce propos Grace A. Gill Mark (1927), Anne-Marie du Boccage: une femme de Lettres au XVIII .

7. Madame Leprince de Beaumont publie son premier traité d'éducation fin 1752 : Éducation complète ou abrégé de l'Histoire universelle, mêlée de géograhie et de chronologie (Kaltz 2000 : ix). Il faut tout de même souligner que dans ses Lettres diverses et critiques (Nancy, 1750) et dans ses Lettres de Madame du Montier à la marquise de *** sa fille, publié par épisodes dans Le Nouveau Magasin Français (1750-1752), elle avance de-ci de-là certaines idées sur l'éducation, en général, et sur celle des filles, en particulier. Ce n'est que longtemps après qu'elle se réferera à ses Lettres diverses et critiques contenant l'« Avis aux Parens \& aux Maîtres » comme à son « Traité d'éducation » (Bloch 2003 : 89). Mais cette appellation ne date pas de 1750, l'année où se sont rencontrées les deux femmes à la réception chez « Mylady Montaigu », la lettre contenant la référence envoyée à sa sœur cadette date du 25 mai 1750. 
Marie du Bocage conçoit le genre épistolaire et ce qu'elle attend de lui, pour ensuite pouvoir apprécier et pouvoir mesurer la portée de la référence élogieuse qu'elle fait à Jeanne-Marie Leprince de Beaumont dès les premières pages de sa correspondance publiée.

\section{Potentiel du genre Épistolaire pour Anne-Marie du Bocage}

Tout d'abord voyons quel potentiel Anne-Marie du Bocage accorde au genre épistolaire. Encouragée par MM. Le Périsse, à la vue d'une œuvre de qualité, cette femme auteur et salonnière (connue et célébrée à son époque, oubliée et ignorée jusqu'à il y a très peu de temps ${ }^{8}$ ), Anne-Marie du Bocage par privilège du roi publie pour la première fois en 1764 dans le recueil de ses œuvres, Les Lettres sur l'Angleterre, la Hollande et l'Italie, et tel qu'elle l'avoue elle-même dans le paratexte du troisième volume ${ }^{9}$, c'est elle qui assume le choix et la décision de la publication. Elle ajoute qu'elle y a apporté les retouches au style, les corrections et les suppressions nécessaires de tout ce qui se rapporte aux questions familiales, puisqu’au départ ces lettres privées n’envisageaient que la première destinataire, ou tout au plus le cercle d'amis le plus proche. Évidemment, des explications et des mises en garde qui nous rappellent sans aucun doute les principes du jeu de modestie et de dissimulation de son époque (Bloch 2000 : 85). Consciente et avisée des enjeux du genre épistolaire, Anne-Marie du Bocage ne fait certes pas mention dans ce paratexte des ajouts en vue de cette publication. Nous en avons donné un exemple avec l'éloge et référence qui nous occupent. Pouvons-nous soupçonner qu'il y en eut bien d'autres sur le texte définitif arrivé aux mains des lecteurs et des lectrices...

Comme nous aurons l'occasion de voir tout au long de notre texte, la justification du geste épistolaire dépasse amplement le caractère communicatif de l'épitre, les lecteurs et lectrices peuvent constater dès le départ que le geste d'écriture l'emporte amplement ${ }^{10}$ et, comme nous l'avons démontré dans un tra-

8. Nous rappelons dans ces lignes l'intéressant colloque international sur Anne-Marie du Bocage qui a été organisé et dirigé par François Bessire et Martine Reid, tenu à Rouen le $1^{\mathrm{er}}$ et le 2 décembre 2011. Un colloque qui a permis à nombre de chercheurs d'apporter de nouvelles lueurs sur cette femme auteur du XVIII ${ }^{e}$ siècle, certes très connue à son époque, et cependant sombrée dans l’oubli depuis très longtemps.

9. La première publication des Lettres sur l'Angleterre, la Hollande et l'Italie remonte à 1764 dans le troisième volume du Recueil des œuvres de Madame du Bocage à Lyon, chez MM. Le Périsse. Elles furent rééditées en 1767 et 1770 , et publiées séparément en 1771. C'est la réédition de 1770 que nous utilisons pour notre étude.

10. Anne-Marie du Bocage est très consciente que l'écriture des lettres assouvit son appel de l'écriture et sa pratique. Nous rappelons les termes de J. K. Holland quant à la pratique épistolaire dans 
vail précédent, les lettres sont l'espace de son propre plaidoyer pour son statut de femme auteur ${ }^{11}$. Pour peu que l'on avance dans cette correspondance, on en vient à observer qu'Anne-Marie du Bocage n'ignore nullement que la pratique du genre épistolaire dans ce XVIII ${ }^{\mathrm{e}}$ siècle présente un potentiel qui va beaucoup plus loin que celui de la communication tel que le rappelle Marie-Laure Girou Swiderski (2009 : 8) pour d’autres femmes auteurs dans son article « La République des lettres au féminin. Femmes et circulation des savoirs ». Les Lettres sur l'Angleterre, la Hollande et l'Italie, de façon devancière et, certes audacieuse, vont s'avérer pour leur auteure l'espace où, parfois de façon très subtile ${ }^{12}$, mais aussi très souvent ouvertement, elle va composer son autoportrait ${ }^{13}$ au fil des jours, de ses voyages, de ses rencontres, de ses réflexions et de ses créations : celui d’une «femme de Lettres » de son temps qui revendique pour elle-même, son statut d'auteur sans que la honte, la pudeur ou la peur du ridicule ne l'en empêchent. Si d'emblée, comme dans bien des correspondances, les lettres semblent à priori prétendre cette communication avec les proches tel que nous l’avons rappelé, le témoignage de ce qui lui arrive au quotidien dans les pays fréquentés, l’expression des diverses réflexions

son œuvre The Letter Form and the French Enlightenment: The Epistolary Paradox : « Almost any kind of prose composition can be found in epistolary form and by the second half of the eighteenth century, the letter has become a kind of all-purpose literary omnibus. It is used for religious and political satire, divine and secular instruction, philosophical essays, literary criticism and detailed first person reports on travel to exotic lands » $(1991: 169)$.

11. Voir à ce propos notre texte « Genre épistolaire et autoportrait. Les lettres sur l'Angleterre, la Hollande et l'Italie comme espace de plaidoyer pour le statut de femme auteur ». (Corbí 2016 : 151-166).

12. «[...] Si je me borne à vous faire mon histoire, notre amitié vous la rendra intéressante; mais vous ennuieriez ceux à qui vous voulez lire mes lettres. Il est vrai que la manie de parler souvent de soi, traitée de vanité en toute autre occasion, ne doit point l'être dans une correspondance dont le seul but est de se communiquer l'une à l'autre les choses qui nous concernent et nous affectent le plus. Tâchons donc, en vous instruisant de ce qui me regarde, de vous amusez des merveilles dont je serai le plus frappée » (Du Bocage 1770 : 125-126).

13. Si l'autoportrait amorce son chemin au XVIe siècle, avec le XVIIe l'autoportrait subit un déclin

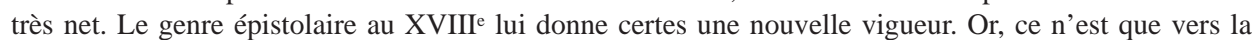
moitié du siècle que les correspondances privées seront publiées du vivant de leurs auteurs tel que nous l'avons rappelé précédemment en citant Janet Gurkin Altman « The Letter Book as a Literary Institution 1539-1789: Toward a Cultural History of Published Correspondences in France », op. cit. En France, le portrait littéraire commence son cheminement au XVI e siècle, rappelons à ce sujet les Essais de Montaigne et le célèbre autoportrait que l'auteur a légué à l'histoire de la culture occidentale, ou bien dans le genre épistolaire le portrait de soi que nous offre Du Pasquier. Au XVIIe siècle le portrait littéraire, genre très prisé dans la société galante, atteint sa maturité avec des auteurs tels que $\mathrm{M}^{\text {lle }}$ de Montpensier et les frères Scudéry, parmi d'autres. Cependant, le Grand Siècle ne peut concéder de place qu’à l'autoportrait fictif, qui permet de façon voilée que le lecteur puisse arriver à soupçonner qui se cache derrière les mots. Dans le genre autobiographique, le sous-genre des Mémoires, très en vogue à partir de l'âge classique, permettra la peinture de soi. Pourtant, les textes qui s’encadrent dans ce groupe n’ont pas été publiés du vivant de leurs auteurs. Voir à ce propos Jacqueline Plantié (1994), La mode du portrait littéraire dans la société mondaine en France (1641-1681). 
qui lui surviennent, voire même « l'instruction » de sa sœur - ce qui retiendra notre attention dans le deuxième versant de notre article -, dans cette « histoire de soi » qu'elle construit et le portrait qu'elle tisse au jour le jour, dans cet « artisanat » de soi que sont les correspondances selon les termes de Bernard Beugnot (1990 : 36), Anne-Marie du Bocage en profite pour retourner dans le passé pour rendre compte au fil des épitres de la très riche formation qui l’a amené à se dévouer aux Lettres, à développer ses goûts esthétiques, et nous commente les œuvres d’art admirées et les monuments visités, nous parle de ses innombrables lectures, de nombreux auteurs, de ses propres œuvres - les précédentes et celles qu'elle vient de créer à l'occasion durant ses itinérances et séjours -, et amène ses lecteurs et lectrices à lire ses vers et ses traductions faits en fonction des circonstances.

Les Lettres qu'elle a composées et qu'elle décide plus tard de publier de son vivant sont celles d'une femme auteur « à l'œuvre » qui côtoie en toute normalité et égalité les philosophes célèbres de son époque. Très subtilement, elle a recours à une série de stratégies pour démontrer combien les grands hommes français et étrangers de son siècle, la tiennent eux-mêmes pour une femme auteur consacrée. Nous retenons ici, comme un élément révélateur, le fait qu’elle transmette, par exemple, d'abord à sa sœur et à ses proches amis, à ceux des réseaux des salons pouvons-nous ajouter, et, ensuite avec cette publication, à ses lecteurs et lectrices, les opinions que les grands lettrés ont versées sur elle, tels que, par exemple, Mylord Chesterfield lors de son séjour en Angleterre qui « la louant à l'envie lui demanda des exemplaires de ses œuvres », le baron de Groningue qui lui récita par cœur des passages des Amazones, le Pape Benoît XIV qui la reçut tel un grand homme lettré, les différentes assemblées qu'elle fréquenta à l’étranger... Or, sans aucun doute, le plus révélateur est Voltaire - le patriarche de Ferney, le Homère français, selon elle - qui, occupant une place prépondérante dans ces lettres, toujours élogieux à son égard, a ajourné son voyage pour la recevoir et lui faire les honneurs aux Délices.

Une histoire personnelle et un autoportrait qui repose davantage sur le besoin de cette « connaissance littéraire » tel que le rappelle Bernard Dray (2000 : x), analysant les épistolières du XVIII e siècle, et du besoin de se présenter, nous l'avons soutenu précédemment, comme femme auteur pour s'inscrire ainsi ellemême dans la postérité. Dans notre étude "Réflexivité et autoréflexivité dans Les Lettres sur l'Angleterre, la Hollande et l'Italie de Mme du Bocage » (Corbí 2017 : 57-68) nous avons pu démontrer que le caractère réflexif de ses textes dévoile bel et bien que l'épistolière conçoit sa correspondance avec sa sœur à l'instar du commerce littéraire qu'elle établit avec ses autres amis les philosophes, et que ces « Volumes » $(1770: 176)$ tiennent plus d'une œuvre littéraire, car l'appel et le geste d'écriture l'emportent largement sur le « geste de com- 
munication » et de témoignage. Ainsi, les voyages et séjours d'Anne-Marie du Bocage lui permettent de disserter sur de multiples thèmes qui sont d'actualité et qui pourraient être résumés sous cette inquiétude anthropocentrique renouvelée à la lueur de la raison, des sciences expérimentales et du vécu humain dans toutes ses dimensions propre au siècle des Lumières. Les témoignages et les réflexions personnelles qu'elle a livrés à la postérité sont à situer, nous le défendons dans notre étude citée antérieurement, dans le cadre du débat d'idées de son époque, et démontrent combien, en son propre nom et à la première personne, elle a voulu prendre part de façon explicite aux controverses de son temps. Dans le cadre de notre article nous retenons d'autant plus notre attention sur le fait que dans ce débat d’idées elle aspire à se ranger indéniablement aux côtés de Madame Leprince de Beaumont tel que nous allons le démontrer dans les lignes suivantes.

\section{Enjeux et portée de la référence à Madame Leprince de Beaumont dans cette correspondance}

Comme nous l'avons avancé précédemment, le caractère réflexif de la correspondance permet d'avoir à l'esprit d'autres œuvres et auteurs interpelés qui agissent comme des miroirs dans lesquels se reflètent les mots et les discours d'Anne-Marie du Bocage. Nous soutenons que cette référence élogieuse à Madame Leprince de Beaumont n’est pas gratuite. Nous défendons que située au tout début de la correspondance, elle interpelle le savoir des lecteurs et des lectrices et qu'elle conditionne leurs lectures. Dans notre introduction nous avons suggéré d'emblée plusieurs facteurs qui pourraient déjà justifier ce compliment introduit à postériori, pourtant il y en a un qui, à notre avis, semble faire le poids. Outre le fait que lors de son séjour en Angleterre, elle ait connu et apprécié de près et sur place la pratique pédagogique de Madame Leprince de Beaumont, outre le fait qu'elle ait contribué avec quelques-uns de ses textes aux premières œuvres de la célèbre pédagogue et gouvernante ${ }^{14}$, Anne-Marie du Bocage, en 1764, connaît indubitablement sa trajectoire littéraire et, tenant compte des réseaux et des circulation des savoirs dans ce siècle, nous pouvons affirmer sans risque, qu'elle

14. Nous tenons à signaler qu'il y a des critiques qui arrivent à affirmer que Leprince de Beaumont fut aidée par Du Bocage pour certains de ses travaux. Voir à ce sujet l' «Introduction » à Femmes savantes et femmes d'esprit de Roland Bonnel et Catherine Rubinger (1994 : 17). « [...] Madame Leprince de Beaumont, on the other hand, in her Nouveau Magasin Français (1750-1752), wrote and published (occasionally assisted by Madame du Bocage) short stories, anecdotes, poetry, memoirs, articles about famous women and women's education, morality and the arts ». Nous soutenons qu'il faudrait pondérer ces affirmations. Aspect que nous aborderons dans une prochaine étude. 
est non seulement au courant de toutes les publications de l'éducatrice rouennaise rencontrée en Angleterre, mais aussi de son succès international qui à cette époque-là est déjà frappant par son ampleur (Kaltz 2000 : xviii-xix).

Effectivement, dans l'histoire personnelle, l'histoire d'une formation à vrai dire, et l'autoportrait qu'Anne-Marie du Bocage construit, ses lecteurs et lectrices peuvent déduire ses capacités intellectuelles, la soif de découvertes, d’apprentissages et de connaissances qui l'ont caractérisée depuis un très jeune âge, le rôle joué par les parents dans son éducation et en particulier celui de son père (1770 : 50). Nous pouvons aussi comprendre pourquoi elle a choisi de se dévouer activement aux Lettres et de fréquenter les grands hommes. Une histoire de soi et un autoportrait qui ne peut manquer de renvoyer au débat sur l'égalité des sexes et du besoin d'éducation des femmes qui, tout le long du XVIII e siècle, mais tout particulièrement à partir de la mi-décennie, a vivement marqué et occupé les milieux intellectuels tel que le rappelle $\mathrm{M}^{\mathrm{a}}$ Angeles Llorca Tonda (Llorca Tonda 2016 : 90).

Considérant les caractéristiques de cette femme exceptionnelle que fut AnneMarie du Bocage, et le compliment et reconnaissance que celle-ci adresse à Jeanne-Marie Leprince de Beaumont au tout début des Lettres sur l'Angleterre, la Hollande et l'Italie, les lecteurs et lectrices complices ne peuvent manquer d'avoir en tête de nombreux textes. Si, effectivement, ceux de Madeleine de Puisieux, par exemple, reviennent à notre esprit, ceux de la pédagogue, également. Entre autres ${ }^{15}$, la « Lettre en réponse à “l’Année merveilleuse” », l’une des toutes premières œuvres de Madame Leprince de Beaumont publiée en $1748{ }^{16}$, et qui critique, comme nous le savons, les arguments utilisés par l'abbé Coyer dans sa justification de l'inégalité des sexes ${ }^{17}$. Pour la future auteure des magasins, ce n'est pas la nature qui rend la femme inférieure à l'homme, c'est bien le manque d'éducation et de formation, la « crasse » ignorance (Beaumont in Kaltz 2000 : 36) qui l’a condamnée à cette condition d’infériorité au grand profit des hommes

15. Pensons aussi à l' " Avis aux parents et aux maîtres, sur l’éducation des enfants », dans Les lettres diverses et critiques (1750) et à certains textes que Mme Leprince de Beaumont publie dans le Nouveau Magasin français (1750-1752).

16. 1748 est également l’année de publication du Triomphe de la vérité.

17. «[...] L’auteur de cet ingénieux ouvrage, mériterait sans doute nos actions de grâce, si le seul amour de la justice lui avait dicté les éloges qu'il nous donne; mais son intention n’était pas de nous obliger, s'il remarque avec soin les caractères distinctifs des deux sexes, c'est pour abaisser le nôtre, qu'il insinue [...] Si l'action est une perfection on ne peut nier que l'oisiveté ne soit un défaut \& qu'un être est d'autant plus parfait qu'il peut agir avec moins d'interruption. Quel avantage n'a donc pas notre langue sur celle des Hommes, puisque naturellement elle peut s’acquitter de son office avec plus de légèreté et de continuité ? [...] C’est par la même raison que les Femmes écrivent mieux que les hommes [...] » (Leprince de Beaumont in Kaltz 2000 : 81-83). 
- «tyrans », selon elle - qui sont en fait les responsables de l'anéantissement de ses capacités intellectuelles. La femme, ainsi, a-t-elle toujours été sous leurs tutelles et n’a pu s’épanouir comme individu. Elle doit passer « d'être parlant, écoutant et regardant » à « être pensant » (Beaumont in Kaltz 2000 : 36) pour parvenir à « bien vivre » (Beaumont in Kaltz 2000 : 36) nous rappelle Beaumont. On pourrait penser à un féminisme avant la lettre ${ }^{18}$. Sans aucun doute, nous semblet-il. Anne-Marie du Bocage, quant à elle, selon l'espace qu'elle concède à son époux dans les lettres publiées de son vivant fait preuve également de ce souhait de détachement de la tutelle et des contraintes maritales infligées par la société19, causes premières de la sous-estimation des capacités intellectuelles des femmes. Accompagnée par lui durant ses voyages, avertissant au tout début qu'il va l'aider à faire ses réflexions, les lecteurs et lectrices des Lettres sur l'Angleterre, la Hollande et l'Italie observent cependant que le mari sur plus de quatre cents pages n'apparaît que cinq fois et de façon très discrète, toujours en arrière-plan, et bien évidemment sur des aspects qui tiennent beaucoup plus du quotidien, donc qui ne se rapportent aucunement à sa vocation et dévouement littéraires ${ }^{20}$.

Pourtant, il nous semble que le jeu intertextuel déclenché par l'éloge d’AnneMarie du Bocage envers Madame Leprince de Beaumont va beaucoup plus loin.

18. Malgré sa foi catholique et ses croyances religieuses, on peut affirmer que la trajectoire vitale de Jeanne-Marie Leprince de Beaumont est déjà en elle-même un exemple d'assomption de son être (sa première demande d'annulation de mariage, son dévouement au travail pour vivre, l'éloignement par rapport au deuxième époux en repartant en France pour rejoindre sa fille, etc.). Ses idées sur l'éducation des femmes sont bien devancières pour l'époque même si la pédagogue voue les femmes au foyer au service du bonheur conjugal et familial. Mais, la question qui ne peut échapper à notre esprit est celle de si à cette époque les femmes pouvaient envisager un autre destin que celui du couvent ou du mariage.

19. Précisément l'espace très restreint dédié à l'époux dans les lettres publiées nous fait soupçonner également que cette question a dû subir des retouches en vue de la publication. Anne-Marie du Bocage annonce à sa sœur au début de sa correspondance que son époux va l'aider à faire les réflexions. N'est-il pas surprenant de constater le peu de place qu'elle lui concède par la suite sur quatre cents pages ?

20. S’il est vrai que la Renaissance avec les valeurs humanistes a défendu l'instruction des femmes comme un complément d'agrément et de réussite à cet ordre social qu'est le mariage rompant dans une certaine mesure avec l'extrême misogynie médiévale, le XVII ${ }^{\mathrm{e}}$ siècle a bien voulu voir la femme mondaine cultivée à l'image de l'honnête homme, mais toujours en liant le sort des femmes aux hommes. Voir à ce sujet l'intéressante étude de Linda Timmermans, L'accès des femmes à la culture (1598-1715) (Paris : Honoré Champion, 1993). C'est vers la fin du XVIIe siècle qu'est repris avec un élan et enthousiasme croissants le débat d'idées sur l'éducation des femmes, pour atteindre un point culminant vers la moitié du siècle suivant. Nombreuses sont les œuvres et les auteurs qui soutiennent que les femmes sont dotées des mêmes potentiels pour l'éducation et l'instruction, et qu'elles ont les mêmes droits que les hommes à participer activement, à la première personne et en leur propre nom dans la vie culturelle, exigeant donc de rompre avec les différentes tutelles, fussent-elles maritales, fussent-elles intellectuelles. Nous citons à ce propos, parmi beaucoup d'autres, les œuvres de Madeleine de Puisieux La femme n'est pas inférieure à l'homme (1750), Le triomphe des dames (1751). 
Précisément, dans ce « commerce littéraire » que permettent les correspondances et les dialogues qu'elles établissent avec les œuvres et les auteurs interpelés, il nous semble que Les lettres sur l'Angleterre, la Hollande et l'Italie en concédant un espace incontournable à l'éducation, renvoient souvent à l'auteure des divers magasins. Nous l'avancions au début de notre texte, les épitres sont l'espace où Anne-Marie du Bocage va se vouer à l' " instruction $»^{21}$ de la sœur cadette, veuve et mère de deux enfants, qui, pleine de curiosité et éloignée du faste et de la vie mondaine, est certes disposée à l'éducation ${ }^{22}$ dans le calme de son château. D'une part, nous retenons qu'au XVIII ${ }^{e}$ siècle, la lettre, comme l'a souvent souligné la critique, remplace la conversation qui ne peut avoir lieu lors des voyages et des déplacements, d'autre part, en transmettant les « apprentissages » par écrit, Anne-Marie du Bocage avertit bien sa sœur de garder les lettres en vue de futurs commentaires. Donc des apprentissages qui ne peuvent être conçus sans le dialogue entre l'éducatrice et l'élève. Comme l'avertit l'épistolière à sa cadette :

[...] Combien faut-il que je vous aime, pour avoir trouvé les moments de tant écrire au milieu des amusements du monde \& des fatigues de la route! Vous voulez payer ma peine en m'assurant que vous vous donnez celle de garder mes lettres: puisque vous prenez ce soin obligeant, nous les commenterons donc ensemble à loisir [...] (1770 : 395).

Une exigence qui nous renvoie au précepte pédagogique du dialogue, fondement de la méthode de Jeanne-Marie Leprince de Beaumont qui s’est appuyée sur les anciens. Parfois même, Anne-Marie du Bocage insiste sur le besoin de s'attacher aux expériences vitales et esthétiques, aux réalités vécues et décrites lors de ses voyages, et surtout aux réflexions ${ }^{23}$ et raisonnements personnels trans-

21. « [...] Si je me borne à vous faire mon histoire, notre amitié vous la rendra intéressante; mais vous ennuieriez ceux à qui vous voulez lire mes lettres. Il est vrai que la manie de parler souvent de soi, traitée de vanité en toute autre occasion, ne doit point l'être dans une correspondance dont le seul but est de se communiquer l'une à l'autre les choses qui nous concernent et nous affectent le plus. Tâchons donc, en vous instruisant de ce qui me regarde, de vous amusez des merveilles dont je serai le plus frappée » (Bocage 1770 : 125-126).

22. Sa formation, sa culture classique et moderne, sa connaissance des langues étrangères, surtout de l'anglais, son plaisir aux lectures tout azimut depuis un très jeune âge, ses voyages, ses rencontres des gens d'esprit, ses amis lettrés des différents Cénacles - français ou étrangers -, ses réflexions et sa morale personnelles, ses traductions et, surtout, ses œuvres établissent une distance très nette entre la femme auteur - éducatrice de vocation - et l'élève, certes, pleine de curiosité.

23. « [...] Cent personnes regardent le même objet \& l'envisagent sous divers points de vue: puisse ma manière de voir, ajouter quelqu'agrément à vos lectures sur les lieux que je suis en train de parcourir » (1770 : 119). 
mis à sa destinataire, qui lui « seront d'un bien plus grand profit », car pour les renseignements plus précis les livres des meilleurs voyageurs reposent sur les étagères des bibliothèques ${ }^{24}$. Mais des apprentissages qui en passant par le dialogue, doivent amener aux raisonnements face à face entre l'éducatrice et l'élève. Impossible de ne pas penser à ce qui, tel que le souligne Alicia Piquer Desvaux, a fait l'originalité de Leprince de Beaumont:

La pédagogue qui connaît et suit Rollin, Fénélon et Locke, affirme que la capacité intellectuelle de la femme est égale et parfois supérieure à celle de l’homme, tout simplement parce que la femme s’inspire de son expérience quotidienne, observe et écoute, afin de bien raisonner (Piquer 2016 : 200-201).

La référence et l'éloge à l'œuvre de du Beaumont déclenche évidemment un jeu intertextuel qui nous amène à commenter certains aspects des Lettres sur l'Angleterre, la Hollande et l'Italie d'A. M. du Boccage qui, nous le soutenons, renvoient aux principes pédagogiques, à la pensée, et à l'œuvre de Jeanne-Marie Leprince de Beaumont.

Si, effectivement, Anne-Marie du Bocage, n’a pas écrit de traité d’éducation, il faut admettre, cependant, qu'elle ne fait pas exception à son siècle, que ce thème l'intéresse vivement et qu'elle veut participer au débat. Ses lettres témoignent de ce fait. De nombreux passages en constituent de vifs exemples. N'ayant pas eu d'enfants, l'amour qu'elle porte envers ses neveux la tient en constante inquiétude au sujet de leur éducation. Voyons un exemple:

[...] Je ferais en vain remarquer à mes neveux combien la moindre mauvaise habitude est dangereuse; les sottises des pères, dit le sage Fontenelle, sont en pure perte pour leur prospérité. Ainsi je me tais \& vais me reposer (1770 : 196).

S’il est vrai que Fontenelle - natif de Rouen et ami de du Bocage, également - est nommé ici, on ne peut pour le moins penser à Jeanne-Marie de Leprince de Beaumont pour diverses raisons. D’une part, parce que le nom de Fontenelle renvoie aux tous premiers collaborateurs du Nouveau Magasin français, d'autre part, parce que l'auteur des Entretiens sur la pluralité des mondes s'érige, comme il est fort

24. «Ceux qui n’ont point vu les objets dont je parle, pourront dans mes faibles esquisses, prendre le désir d'en chercher des portraits plus frappants dans les meilleurs voyageurs ; \& ceux qui les connaissent par eux-mêmes, ne seront peut-être pas fâchés de se les rappeler avec moi [...] ». " Avertissement » (Du Bocage 1770 : Aij). 
connu, comme figure phare dans une première conception multidisciplinaire de l’éducation. Madame Leprince de Beaumont, précisément, a été pionnière pour l'éducation des filles, grâce entre autres, au recours dans le cadre de sa pratique et de ses traités à diverses disciplines ${ }^{25}$ qui auparavant ne visaient que l'éducation des garçons. Finalement, « cette sottises des pères » ne peut que nous faire penser à l'importance que les parents tiennent dans la formation de leurs enfants, une prémisse qui a été constamment soutenue par Jeanne-Marie Leprince de Beaumont dans ses efforts pour changer les préceptes éducateurs vieillis, et absolument néfastes pour le bon fonctionnement de la société.

Lors de son séjour en Angleterre, en pleine admiration, Anne-Marie du Bocage ne manque pas de vanter à sa première destinataire et plus tard à ses lecteurs et lectrices les vertus de la société anglaise - beaucoup plus avancée que la française. En décrivant les mœurs du pays visité, et plus particulièrement le rôle joué par les femmes dans l'éducation de leurs enfants, l'épistolière interpelle d'autant plus, à notre avis, les textes de la pédagogue, certes, parmi beaucoup d'autres.

Les mères anglaises sont convaincues du besoin de spontanéité et de joies des enfants, et se dévouent à leur éducation en toute simplicité et naturel, en les éloignant de l'apparat des cercles mondains, nous dit Anne-Marie du Bocage (1770 : 38). Elles sont vigilantes du respect des convictions familiales. Évidemment, l'épistolière remet en question toute l’éducation française laissée aux mains des pensionnats pour les garçons et des sombres couvents pour les filles. Avec ces mots, il est difficile, pour les lecteurs et lectrices complices, de ne pas avoir en tête la pensée de Madame Leprince de Beaumont exprimée de façon préliminaire dans son « Avis aux parents et aux maîtres, sur l'éducation des enfants » publié dans les Lettres diverses et critiques (1750), et développée par la suite dans ses manuels. Dans ce texte comme dans nombre de ses œuvres postérieures, elle s’en prend au manque de vocation et de talent pour l'enseignement de la part des religieuses qui traitent les enfants, et en particulier les filles, tels des « marmottes » (Beaumont in Kaltz 2000 : 89). Ce sont les parents, et en particulier les mères, aidées ou non de gouvernantes qui doivent surveiller de près l'éducation de leurs enfants.

Ces mères anglaises, assument le passage du temps et n’ont pas peur de se montrer « ridées » en public. Vigilantes de l'éducation de leurs filles et du respect des valeurs de leur milieu, elles les accompagnent elles-mêmes en société. Rien à voir avec ce qui se passe dans la société française où les mères sont beaucoup

25. Il s’agit d’Histoire, de Géographie, de Physique, de Sciences Naturelles, de Littérature, de Mathématiques, de Religion, de Français Langue Étrangère, de Morale, etc. 
plus portées sur la frivolité, la coquetterie, sur ce besoin irrépressible de plaire en société - un besoin irrépressible car acquis depuis l'enfance -, et non sur l'intérêt de leur famille, nous dit Anne-Marie du Bocage (1770 : 39). Rejetant l'« ennui » auquel succombent les françaises, ces femmes anglaises, affirme l'épistolière, occupent leur temps dans les plaisirs d'une vie simple, dédiée à leur famille et en étroit contact avec la nature, parfois en société avec leurs amis, ou se vouant à la lecture et à l'étude. La femme, Madame Leprince de Beaumont l'a souvent soutenu, soit dans ses textes non fictionnels soit à travers la fiction ${ }^{26}$, doit se plaire à lire et à s'instruire, ainsi pourra-t-elle prendre elle-même en charge ou surveiller de près l'éducation de ses enfants et rendre ses proches plus heureux. L'instruction de la femme vise cet « être pensant » dont nous parlions précédemment, un être pensant qui lit « pour mieux vivre ». Jeanne-Marie Leprince de Beaumont signale dans le Magasin des adolescentes l'utilité de la femme instruite pour la société. Nous retenons ses mots :

[...] Mais il faut penser à former une fille de quinze ans une femme chrétienne, une épouse aimable, une mère tendre, une économe attentive, un membre de la société qui puisse augmenter l'utilité et l'agrément (Leprince de Beaumont 1817 : 235).

En fait, pour notre épistolière, ces mères anglaises sont bien l'exemple de femmes qui refusant la vanité de la mondanité, cherchent à donner un sens à leur vie avec ce dévouement envers leurs enfants et leur famille, avec les plaisirs simples fondés sur l'éducation et la culture, source de vertu et de sagesse qu'elles transmettent également à leurs filles (1770: 64). L’Éducation et la culture doivent être au centre de la vie des femmes pour Anne-Marie du Bocage. En fait, l'épistolière aborde bien cette morale de vie qui interpelle à plusieurs reprises, nous semble-t-il, les œuvres et la pratique de Jeanne-Marie Leprince de Beaumont.

\section{En guise de conclusion}

L'étude et la culture comme morale de vie que partagent Anne-Marie du Bocage et Leprince de Beaumont nous amènent plus loin à notre avis. Nous voyons une plus grande portée à cette référence. Nous avons soutenu dans les pages précédentes que Les lettres sur l'Angleterre, la Hollande et l'Italie sont l'espace de son propre plaidoyer comme femme auteur. Avec elles, elle a laissé à la postérité son image de

26. Voir, par exemple, le conte Bellotte et laideronette dans le Magasin des enfants (Leprince de Beaumont 1817 : 356-366). 
soi à l'œuvre. Et c'est dans cette image de soi à l'œuvre que la référence à Madame Leprince de Beaumont acquiert tout son sens et son poids, nous semble-t-il. Dans cette participation au débat d'idées de son siècle, et en particulier dans la question du besoin d'éducation et de culture chez la femme en toute égalité avec l’homme, ayant interpellé fondamentalement de nombreux auteurs hommes, mais en incluant également la célèbre éducatrice, nous pouvons voir une double intention : d'une part, rendre un hommage certain à cette « gouvernante de l'humanité toute entière » selon Alix Deguise (1994 : 179), et à l'aide de ses lettres publiées de son vivant contribuer à cette part de postérité qui, en toute justice, lui est incontestablement due. D'autre part, dans ce jeu de miroirs que constitue le travail intertextuel de tout texte, par le biais de cette référence élogieuse, il nous a semblé que nous pouvons y voir chez Anne-Marie du Bocage une prise de position courageuse ${ }^{27}$ à son époque pour la défense de la vocation des lettres pour les femmes, et en cela elle rappelle les mots célèbres de la directrice du Nouveau Magasin français qui déjà en 1750 se définissait audacieusement comme une « auteur femelle » en rejetant le rôle « de noueuse de ruban » assignée par la société de son temps.

Finalement, avec cet éloge à Jeanne-Marie Leprince de Beaumont, il nous semble que dans ses Lettres sur l'Angleterre, la Hollande et l'Italie Anne-Marie du Bocage tient à se situer aux côtés de la célèbre pédagogue avant la lettre et à revendiquer leur place de droit à leur époque et pour l'avenir dans les vifs débats sur l'Éducation qui marquèrent très particulièrement le XVIII siècle français, mais dont les Histoires littéraires et celles des idées philosophiques n’ont retenu que des noms masculins fort malheureusement et injustement jusqu'à très récemment ${ }^{28}$.

\section{Références}

ARTIGAS-MENANT, G. (1991). « La vulgarisation scientifique dans Le Nouveau Magasin français de Mme Leprince de Beaumont ». Revue d'Histoire des Sciences 44 (3) : 343-357.

ARTIGAS-MENANT, G. (2004). « Les Lumières de Marie Leprince de Beaumont: nouvelles données biographiques ». Dix-huitième siècle 36 : 291-301.

27. « Courageuse », disons-nous, car tel que souligné précédemment dans notre texte le statut de « femme auteur » à l'époque d'Anne-Marie du Bocage fut absolument controversé.

28. Voir à ce sujet l'intéressant volume dirigé par Martine Reid Les femmes dans la critique et l'histoire littéraire (2011). 
BEUGNOT, B. (1990). " De l'invention épistolaire à l'invention de soi » en L'épistolarité à travers les siècles. Geste de communication et/ou d'écriture. (Coords. Mireille Bossis y Charles Porter). Stuttgart : Franz Steiner : 27-38. BLOCH, J. (2000). " The Eighteenth century: women writing, women learning » en A history of women writing in France. (Coord. Sonia Stephens). Cambridge : Press Syndicate of the University of Cambridge : 85-101.

BONNEL, R. y RUBINGER, C. (1994). «Introduction » en Femmes savantes et femmes d'esprit. (Coords. Roland Bonnel y Catherine Rubinger). Bern/New York : Peter Lang : 3-43.

CORBÍ SÁEZ, M ${ }^{a}$ I. (2016). « Genre épistolaire et autoportrait : Les lettres sur l'Angleterre, la Hollande et l'Italie comme espace de plaidoyer pour le statut de femme auteur " en Femmes auteur du dix-huitième siècle. Nouvelles approches critiques. (Coords. A. Sirvent Ramos, $\mathrm{M}^{\mathrm{a}}$ Isabel Corbí Sáez et $\mathrm{M}^{\mathrm{a}}$ Angeles Llorca Tonda). Paris : Honoré Champion : 151-166.

CORBÍ SÁEZ, M ${ }^{\mathrm{a}}$ I. (2017). "Réflexivité et autoréflexivité dans Les Lettres sur l'Angleterre, la Hollande et l'Italie de Mme du Bocage » en Forma Venus, arte Minerva. Sur l'œuvre et la carrière d'Anne-Marie du Bocage (1710-1802). (Coords. François Bessire et Martine Reid). Rouen : Presses Universitaires de Rouen et du Havre : 57-68.

DRAY, B. (2000). « Avant propos » en Femmes en toutes lettres. Les épistolières

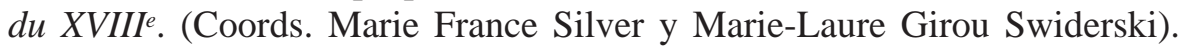
Oxford: Voltaire Foundation : ix-xii.

DU BOCAGE, A.-M. 1770 (1764). Les lettres sur l'Angleterre, la Hollande et l'Italie, Recueil des Euvres. Lyon: MM. Périsse, vol. 3.

GIROU SWIDERSKI, M.-L. (2009). " La République des Lettres au féminin. Femmes et circulation des savoirs au XVIII" siècle ». Canadian Society for Eighteenth-Century Studies 28 : 1-28.

GOOSSENS, K. (2015). " Jeanne-Marie Leprince de Beaumont et son mode d'enseignement pour jeunes filles nobles dans les Magasin des Enfants et des Adolescentes ». @nalyses 10 (1 hiver) : 11-34. <www.revue-analyses. org> (consulté le 15 décembre 2015).

GURKIN ALTMAN, J. (1986). « The Letter Book as a Literary Institution 15391789: Toward a Cultural History of Published Correspondences in France ». Yale French Studies 71 : 17-62.

GURKIN ALTMAN, J. (1992). « Espace public, espace privé : la politique de publication des lettres sous l'Ancien Régime ». Revue Belge de Philologie et d'Histoire 70 : 607-623.

HOLLAND, J. W. (1991). The Letter Form and the French Enlightenment: The Epistolary Paradox. New York: Peter Lang. 
IVERSON, J. y PIERETTI, P. (2004). " "Trois personnes seront admises à concourir”. La participation des femmes aux concours académiques ». Dix-huitième siècle 36 : 313-332.

LEPRINCE DE BEAUMONT, J.-M. 1859 (1756). Le Magasin des enfants, ou Dialogues d'une sage gouvernante avec ses élèves de la première distinction, dans lesquels on fait penser, parler, agir les jeunes gens suivant le génie, le tempérament et les inclinations de chacun. Lyon: Jean-Baptiste Réguilliat.

LEPRINCE DE BEAUMONT, J.-M. 1817 (1760). Le magasin des adolescentes, ou dialogues entre une sage gouvernante et plusieurs de ses élèves de la première distinction. Avignon : Librairie Étienne Chaillot Aîné, vol. II.

LEPRINCE DE BEAUMONT, J.-M. (2000). Contes et autres écrits. Édition présentée par Barbara Kaltz. Oxford : Voltaire Foundation.

LLORCA TONDA, Mª.Á. (2016). « De l'éducation des femmes chez quelques femmes auteurs du XVIII" » en Femmes auteur du dix-huitième siècle. Nouvelles approches critiques. (Coords. A. Sirvent Ramos, $\mathrm{M}^{\mathrm{a}}$ Isabel Corbí Sáez et $\mathrm{M}^{\mathrm{a}} \mathrm{A}$. Llorca Tonda). Paris : Honoré Champion : 89-104.

REID, M. (2007). «Introduction » en Stéphanie Félicité de Genlis, La femme auteur. (Éd. Martine Reid). Paris: Gallimard, coll. « Folio » : 7-13.

REID, M. (2011). Les femmes dans la critique et l'histoire littéraire. (Sous la direction de Martine Reid). Paris : Honoré Champion.

ROBAIN, J.-M. (2004). Madame Leprince de Beaumont intime. Avec ses principaux contes et des documents inédits. Genève: Slatkine.

PIQUER DESVAUX, A. (2016). « Jeanne-Marie Leprince de Beaumont: l'art de bien apprendre à lire et à vivre » en Femmes auteur du dix-huitième siècle. Nouvelles approches critiques. (Coords. de A. Sirvent Ramos, $\mathrm{M}^{\mathrm{a}}$ Isabel Corbí Sáez et $\mathrm{M}^{\mathrm{a}} \mathrm{A}$. Llorca Tonda). Paris : Honoré Champion : 197-208.

PLANTIÉ, J. (1994). La mode du portrait littéraire dans la société mondaine en France (1641-1681). Paris : Honoré Champion. 